\title{
Effects of selective and non-selective NSAIDs on in vitro contractility of the equine jejunum
}

\author{
Anna Wogatzki', Klaus Hopster², Karen Tappenbeck³, Korinna Huber³ and Anna K. Rötting² \\ 1 Justus Liebig University of Giessen \\ 2 University of veterinary medicine Hannover, Foundation; clinic for horses \\ 3 University of veterinary medicine Hannover, Foundation; institute of physiology
}

\begin{abstract}
Summary: NSAIDs are commonly used in colic therapy. Especially in the post-operative stage, dysmotility is a severe complication. Literature provides evidence of an effect on the contractility of the gastrointestinal tract through NSAIDs. Therefore, the aim of this study was to investigate the effects of NSAIDs on the equine small intestine and, in particular, to accurately examine the effects on the injured intestine. Included were horses with a healthy intestine which had been subject to a median laparotomy with two consecutive experimental ischemias and reperfusions. In the first experiment, an in vitro application of the NSAIDs flunixin and firocoxib was carried out. In the second experiment, the respective NSAID was administered to the probands in vivo and the samples were examined by means of a measurement of the contractility in vitro. The in vitro application of the non-selective COX inhibitor flunixin had significant inhibitory effects on the contractility of the equine jejunum in vitro. These inhibitory effects occurred in comparison to all other samples. In vitro application of firocoxib had no significant effects on the contractility in vitro but showed a slight insignificant increasing effect, especially on the frequency of contractions of the circular musculature. After intravenous in vivo application, subsequent sample taking and examination, the results of the measurements of contractility in vitro were clearly more heterogenous. This, for example, led to a significant increase in frequency of contractions of the longitudinal musculature of the samples, damaged by ischemia and reperfusion, after intravenous application of flunixin, and to a significantly higher amplitude of contractions of the longitudinal musculature of the samples, damaged by ischemia and reperfusion, after intravenous application of firocoxib. These inconsistent effects and the deviations to the effects after in vitro application probably indicate a toxicological effect after in vitro application of the agents and an altered initial situation in the second experiment due to a panenteric occurrence (resulting from previous damage through the first experiment). The explanation would be an overdosage and, therefore, an intoxication of the tissue after in vitro application of the agents because the inhibitory effects of flunixin only occurred in high concentrations. Based on the examinations of the current study, significant differences between the in vitro application of flunixin and firocoxib on the in vitro contractility of the equine jejunum could be determined. The inhibitory effects of flunixin were dependent on its concentration and only appeared in high concentrations $\left(2.7 \times 10^{-5} \mathrm{M}\right)$. Although the dosages applied in vitro, were oriented towards plasma concentrations of the agents after intravenous application or concentrations used in other studies, it was not clear whether these concentrations corresponded to the actually reached tissue concentrations after intravenous or oral application of the agents. Due to high plasma protein bindings, the actual tissue concentration after intravenous or oral application could be much lower. In the current study, also merely the in vitro contractility was examined and conclusions regarding in vivo motility could not be drawn absolutely. The clinical relevance of the inhibitory effect of flunixin on the contractility in vitro after in vitro application cannot be estimated due to the current study. Also it is not clear to which extent the type of application (in vitro application and intravenous in vivo application) actually had an influence on the results. In the future, studies have to follow that examine the effects of selective and non-selective NSAIDs on the contractility and motility of the equine intestine after intravenous in vivo application, in order to support the choice of an adequate NSAID, especially in post-operative therapy after colic surgery.
\end{abstract}

Keywords: NSAID, firocoxib, flunixine, colic, colic, therapy, intestine, equine, jejunum, contractility, measurement, analgetics, COX-inhibtors, cyclooxygenase, contractility, small intestine

Zitation: Wogatzki A., Hopster K., Tappenbeck K., Huber K., Rötting A. K. (2016) Effects of selective and non-selective NSAIDs on in vitro contractility of equine jejunum. Pferdeheilkunde 33, 538-547; DOI 10.21836/PEM20170602

Korrespondenz: Anna Wogatzki, Oberaulaerstrasse 2, 34639 Schwarzenborn, Germany; anna.wogatzki@vetmed.uni-giessen.de

\section{Introduction}

Based on different studies, an effect of NSAIDs on the contractility of the gastrointestinal tract seems probable in vitro (Menozzi et al. 2009, van Hoogmoed et al. 1999, 2000 and 2002). In vitro, it seems that there is a more inhibitory effect on the contractility through application of NSAIDs. In the literature, several studies about contractility can be found that examined different intestinal segments. In a study by Menozzi et al. from 2009, the effect of selective and non-selective COX inhibitors on the contractility of the small intestine was examined. Therefore, strips of tissue of the ileum were observed in organ baths in vitro. The non-selective NSAIDs showed no significant effects on the contractility up to a concentration of $0.1 \mathrm{M}$; only higher concentrations of flunixin meglumine showed a significant reduction of the tonic contraction. Neither flunixin meglumine nor indomethacin significantly changed the amplitude of the contraction waves. The COX-2 selective inhibitors, however, showed a dose-dependent inhibition of the tonic contraction as well as of the spontaneous motility amplitude. As possible reasons for this result the authors discussed a rather limited role of the prostaglandins in controlling the equine intestinal contractility in vitro because adding prostaglandins (PG) in vitro achieved no effect. The different measurement results regarding non-selective NSAIDs could be based on direct interaction of the agents, independent of COX inhibition, with the contractile unit. The inhibitory effects of the COX-2 selective NSAIDs could be a result of a participation of the COX-2 isoform in equine intestinal motility (Menozzi et al. 2009). 
Adding flunixin meglumine in vitro to muscle samples of the dorsal and ventral colon as well as the pelvic flexure resulted in a reduction of contractile force in one study. The effect varied in its intensity concerning the circular and longitudinal muscle layer, depending on the localization in the colon (van Hoogmoed et al. 2000). In these studies, other non-selective NSAIDs also induced a concentration-dependent reduction of the contractile activity in relation to the values of the basal frequency in the dorsal colon, ventral colon and the pelvic flexure (van Hoogmoed et al. 1999, 2000).

COX-2 preferential NSAIDs and non-selective COX inhibitors had a dose-dependent inhibitory effect on the in vitro contractility of the ventral and dorsal colon in a further study (van Hoogmoed et al. 2002). In the same study, the COX-2 selective etodolac showed an inhibitory effect on the intestinal musculature in vitro only in high concentrations. Here etodolac had a significantly lower inhibitory effect on the contractility than nabumetone and indomethacin.

According to clinical experiences, obstipations of the large intestine in equine patients occur more often after NSAID application (Ross et al. 1985). Newer study results are contradictory to these: through inhibition of cyclooxygenase the synthesis of prostaglandins, which could play a role in the pathogenesis of the paralytic ileus, was clearly reduced. Therefore, the administration of NSAIDs could enhance, respectively maintain, the motility of the gastrointestinal tract through reduction of the synthesis of inflammatory mediators which inhibit the smooth muscles' contractility (Hardy and Rakestraw 2002). Based on study results, influencing of the intestinal contractility in horses is possible by NSAIDs and especially of large interest in the postoperative phase of equine colic patients due to motility disorders. Most studies deal with the effects of ischemia and reperfusion on the intestinal mucosa, only few studies examine the effect on the musculature.

Through immunohistological examinations of the experimental group of Franz (2013), an expression of cyclooxygenase (COX) COX- 1 and COX-2 in the tunica muscularis and tunica serosa of the equine jejunum could be proven. Damage through ischemia and reperfusion caused increased expression of COX-2 (Franz 2013). It was especially apparent here that this expression was clearly obvious in the longitudinal muscle layer and there especially in muscle cells which were oriented towards the tunica serosa (Franz 2013). This varying expression is especially then interesting when thinking about the various affinity to the COX isoforms of the different NSAIDs.

The aim of this study was to evaluate the effects of selective and non-selective nonsteroidal antiphlogistics on the in vitro contractility of equine jejunum. Thereby, the longitudinal and the circular muscle layer should be examined separately. Especially the effects on intestine, damaged by ischemia and reperfusion, should be monitored because NSAIDs are used especially in colic therapy where these kinds of damages are found often.

\section{Materials and Methods}

The group of probands consisted of 13 horses with a clinically healthy intestine. For the realisation of the experiment, the horses were placed under general anaesthesia following a standardized anaesthesia protocol (xylazine-balanced isoflurane inhalation anaesthesia). The horses were positioned for a median laparotomy. After opening the abdominal cavity, the middle section of the jejunum, between plica duodenocolica and plica ileocaecalis was exteriorized. The experiments occurred immediately following each other in one surgical procedure. After realisation of the experiment, the horses were euthanized free of pain, without regaining conscience.

\section{Ischemia and reperfusion model}

The study is divided into two consecutive experiments.

\section{Experiment 1}

After extracting the control sample from the area of the middle section of the jejunum (a $25 \mathrm{~cm}$ long jejunum segment, after applying ligatures to the providing vessels in the mesentery, in toto), an experimental partial ischemia of 90 minutes was applied. For this, an intestinal loop of the middle jejunum of approximately $50 \mathrm{~cm}$ was ligated with a Bühnerband. The ligature included both intestinal tubes as well as the providing vessels. A warm ischemia with a remaining blood flow of approximately $10 \%$ was intended. The following figures (Fig. 1 and 2) demonstrate the experimental ischemia and the resulting changes of the intestine. A control of the grade of ischemia as well as the following reperfusion occurred by pulse oximetry and $\mathrm{O}_{2} \mathrm{C}$ device (company LEA Medizintechnik, Giessen, Germany). Following the ischemia phase, a reperfusion phase of 30 minutes with subsequent sampling took place by releasing the ligatures. The samples were freed of

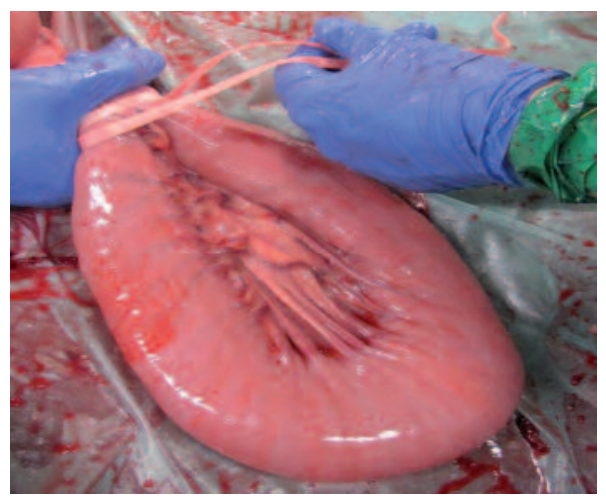

Fig. 1 Creation of the experimental partial ischemia by applying a Bühnerband

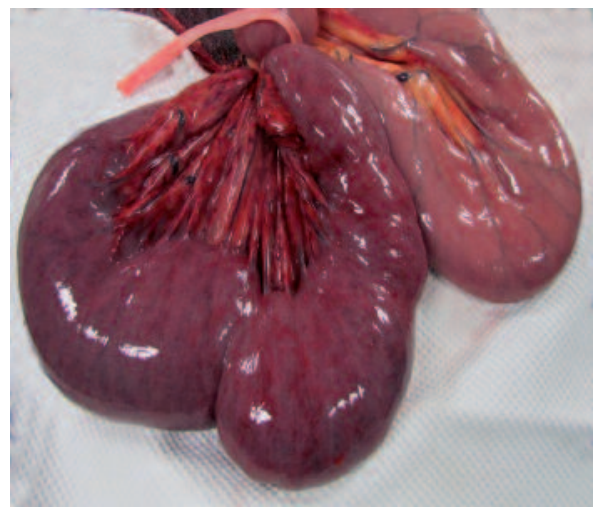

Fig. 2 Intestinal segment after 90 minutes of ischemia 
ingesta by careful rinsing with warm isotonic saline solution and stored in oxygenated Krebs-Henseleit buffer solution.

\section{Experiment 2}

Another extraction of an unharmed intestinal segment (K2) of the jejunum distal to the experimental ischemia and reperfusion occurred with a distance of $100 \mathrm{~cm}$ with the same kind of sample and the same sample preparation. Another experimental ischemia was created in a not-immediately adjacent intestinal segment of the middle jejunum, according to the model described above. After an ischemia over a period of one hour, the probands, after randomization, received one of the both NSAIDs (flunixin meglumine or firocoxib) as intravenous application via peripheral venous catheter in the highest dosage given by the manufacturer (flunixin meglumine 1.1 $\mathrm{mg} / \mathrm{kg}$ Flunidol $^{\circledR}$ RP $50 \mathrm{mg} / \mathrm{ml}$, CP-Pharma Handelsgesellschaft $\mathrm{mbH}$, Burgdorf, Germany, firocoxib $0.9 \mathrm{mg} / \mathrm{kg}$ Equioxx ${ }^{\circledR} 20 \mathrm{mg} / \mathrm{ml}$ Merial, Lyon, France). After expiration of the ischemia time of 90 minutes, the Bühnerband was released and the reperfusion time of 30 minutes was induced. Once more samples were taken of the intestinal segment (R2), damaged by ischemia and reperfusion. Further, immediately after extraction of R2, another sample was drawn of an unharmed intestinal section $100 \mathrm{~cm}$ distal to the experimental ischemia damage (K3). The two-piece setup of the study is graphically depicted in Fig. 3.

\section{Sample preparation}

In order to receive muscle probes, the mucosa with shares of serosa was removed. Muscle strips were cut from the longitudinal and circular musculature. The size of the strips was $1 \mathrm{~cm}$ at length and $0.5 \mathrm{~cm}$ at width. After preparation, thread was tied to the ends of the muscle strips in order to allow better fixation later in tissue chambers and to transducers. The used device consisted of 16 chambers, divided into two systems with eight chambers each, filled with Krebs-Henseleit buffer solution. A continuous fumigation with oxygen was maintained throughout the experiment in order to secure oxygenation of the samples. The thread tied to the upper part of the muscle strip was attached to an adjustable transducer.

The transducer measured the spontaneous contractions (frequency, amplitude, median force) and forwarded these to a measuring unit (Spider 8 chart recorder $4.8 \mathrm{kHz} / \mathrm{DC}$ ) where the data was transformed (Catman Easy software) and displayed as graphics on the monitor. An equilibration time of 60 minutes followed during which in the first 30 minutes the muscle strips were brought to a pretension of $2 \mathrm{~g}$.

\section{Experiment execution}

Feeding of the chambers occurred equally with longitudinal and circular muscle strips.

\section{Experiment 1}

Depending on the type of muscle, two chambers with control samples (K1), two with ischemia and reperfusion samples
(R1), two ischemia and reperfusion samples with flunixin application $\left(R 1_{\text {Flun }}\right)$ and two damaged samples with firocoxib application $\left(R 1_{\text {Firo }}\right)$. The application of NSAIDs occurred in cumulative increase with the concentration stages depicted in Figure 4 with 15 minutes impact time. A continuous measurement of spontaneous contractions followed. Figure 4 graphically shows the time lapse of the first experiment (Fig. 4).

\section{Experiment 2}

Examination after NSAID application in vivo. Here also an equal distribution of the chambers to the muscle strips took place. Thereby, per muscle layer two chambers each had samples without NSAID application as time control (K2), two each had unharmed samples with NSAID application $\left(K 3_{\text {Firo }} / \mathrm{K}_{\text {Flun }}\right)$ and four chambers each had samples with NSAID application, damaged by ischemia and reperfusion $\left(R 2_{\text {Flun }} / R 2_{\text {Firo }}\right)$. The measurement continuously took place as in experiment 1 .

\section{Evaluation and statistics}

Experiment 1

In the evaluation, the contractions after equilibration time and subsequently before the particular NSAID applications at time periods of 15 minutes were observed. Time spans of three minutes each were evaluated, whereas the first time span directly before the first application was used for evaluation. Six time spans of three minutes each were evaluated. The points in time for evaluation were chosen to be after the maximal impact time of the particular concentration stage of the NSAID.

\section{Experiment 2}

Here the contractions were observed in the same time spans as in experiment 1. Likewise, time spans of three minutes

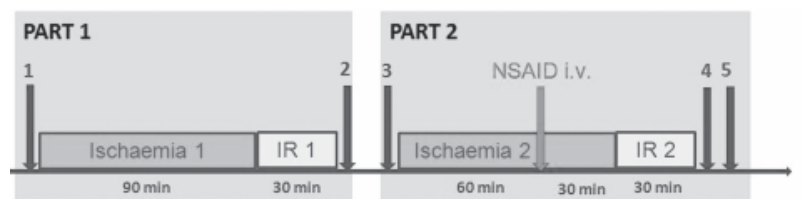

Fig. 3 Depiction of sample taking for both experiments. The samples were taken after each other within one surgery. First experiment: 1. Unharmed sample as control (K1), 2. Sample damaged by ischemia and reperfusion (R1). Second experiment: 3. Unharmed sample for time control (K2), 4. Sample with NSAID damaged by ischemia and reperfusion (R2), 5. Unharmed sample with NSAID (K3)

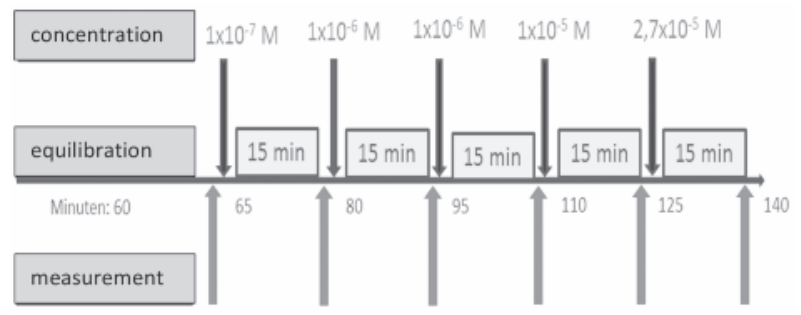

Fig. 4 Depiction of the concentration stages, the temporal sequence of NSAID applications and the time points for measurement (ZP). 
each were examined. In the results, the evaluation of the first and the last time span (measurement 1 and 6) of the experiment is depicted because no significant changes occurred over the course of time.

The statistical calculation of data took place with the statistical program SAS Version 9.3 (SAS Institute, Cary, NC, USA). For evaluation of the linear model the "mixed procedure" under consideration of a significance level of $\leq 0.05$ was cho$\operatorname{sen}(=5 \%)$.

The model residues of the different treatments were tested with Shapiro-Wilks concerning standard distribution and compared with the Tukey-Kramer method after two-factor comparison. The examination within one treatment group over time occurred by Wilcoxon ranked sum test. The graphical depiction of the means and their standard deviation took place with the graphics program Graphpad Prism 5 (Graph Pad Software Inc.) in form of bar graphs as well as Excel (Microsoft Corporation) in form of line graphs.

\section{Results}

Of the twelve remaining probands (one died under general anaesthesia), eleven horses could be used for the in vitro motility measurement (the samples of one proband showed no spontaneous contractility). Muscle strips that showed no contractility during equilibration time, also subsequently developed no spontaneous contractility. In general, the contractions of the strips of longitudinal muscle were weaker than those of the strips of circular muscle. This relates to all three measured parameters: frequency, amplitude and median force.

\section{Effects on the longitudinal muscle after in vitro application}

Effect on the frequency

The damage through experimental ischemia and reperfusion has a significant influence on the frequency of contractions. In the samples $R 1$ as well as $R 1_{\text {Flun }}$ and $R 1_{\text {Firo }}$ a significant increase of the frequency of contractions could be seen in comparison to the control samples (K1) $(\mathrm{P}<0.0001)$. The application of flunixin and firocoxib showed no significant changes in comparison to the untreated samples damaged by ischemia and reperfusion (R1). Especially, there were no significant differences between the samples treated with firocoxib and flunixin $\left(R 1_{\text {Flun }}, R 1_{\text {Firo }}\right)$. Also the cumulative increase of the agent's concentration had no significant effect on the frequency of contractions.

\section{Effect on the amplitude}

The application of flunixin in its highest concentration showed a significant reduction of the amplitude of contractions in comparison to the untreated samples damaged through ischemia and reperfusion ( $R 1) P=0.0281$. There were no significant differences between the samples treated with firocoxib and flunixin $\left(R 1_{\text {Flun' }} R 1_{\text {Firo }}\right)$. The graphical depiction is shown in Figure 5.

\section{Effect on the force}

At the points in time ZP2, ZP5 and ZP6, a significant increase of the median force could be seen in the R 1 samples in comparison to the control samples K1 (ZP2: $P=0.0065, Z P 5$ : $P=0.0123, Z P 6: P=0.0082)$. There were no significant differences between the samples treated with firocoxib and flunixin $\left(R 1_{\text {Flun, }} R 1_{\text {Firo }}\right)$. The cumulative increase of the agent's concentrations only had a significant effect on the median force of contraction in case of flunixin, at the points ZP5 and ZP6 the R1Flun sample showed a significantly lower median force of contractions than the untreated sample $R 1$ at times ZP5 ( $P=0.0418)$ and ZP6 $(P=0.0025)$.

\section{Effects on the circular muscle after in vitro application}

\section{Effect on the frequency}

The damage through experimental ischemia and reperfusion has a significant effect on the frequency. In the samples R1, $R 1_{\text {Flun }}$ and $R 1_{\text {Firo }}$ a significant increase of the frequency of contractions could be seen in comparison to the control samples (K1). Samples treated with flunixin at the highest concentration showed a significantly lower frequency of contractions in comparison to untreated $R 1$ samples and $R 1$ samples treated with firocoxib, $R 1(P=0.0001)$ and $R 1_{\text {Firo }}(P<0.0001)$. Also a change within the samples $R 1_{\text {Flun }}$ could be seen over time. A significant decrease in frequency occurred over time: from ZP2 to ZP6 ( $P=0.0437)$, from ZP3 to ZP6 $(P=0.0006)$, from ZP4 to ZP6 $(P=0.0021)$ and from ZP5 to ZP6 $(P=0.0021)$. In case of application of firocoxib, significant changes could be seen over time. The frequency from ZP5 to ZP6 was significantly higher than at ZP1 (ZP5: 0.0179, ZP6: $\mathrm{P}=0.0014)$.

\section{Effect on the amplitude}

Ischemia and reperfusion had no influence on the amplitude of contractions of the circular muscles. Within the samples $R 1_{\text {Firo }}$ and $R 1_{\text {Flun' }}$ significant decreases of the amplitude of contractions could be seen over time/when increasing the concentrations of the depending NSAID. A significant decrease of the amplitude of the samples $R 1_{\text {Flun }}$ could be seen over time: from $Z P 1$ to $Z P 5(P=0.0018)$, from $Z P 1$ to $Z P 6$ $(P<0.0001)$. A significant decrease of the amplitude of contractions in the samples $R 1_{\text {Firo }}$ could be seen from the time of $Z P 1$ to ZP6 ( $P=0.0163)$. After application of the highest concentration of flunixin, a significant decrease in the amplitude of contractions occurred in comparison to all other samples. The amplitude of contractions of the samples $R 1_{\text {Flun }}$ during the measuring times ZP5 and ZP6 (after 120 and 135 minutes) was significantly lower than those of the samples R1 (ZP5: $\mathrm{P}=0.0459$, ZP6: $\mathrm{P}<0.0001$ ) and at ZP6 also significantly lower than the samples $R 1_{\text {Firo }}(P=0.0041)$ and $K 1$ $(P=0.0070)$.

\section{Effect on the force}

Damage through experimental ischemia and reperfusion had a significant effect on the median force of contractions, a sig- 
nificant increase of the median force of the $R 1$ samples could be seen in comparison to the control samples $\mathrm{K} 1$ starting at ZP3 (ZP3 ( $P=0.0182), Z P 4(P<0.0001), Z P 5(P=0.0020)$ and ZP6 $(P=0.0206)(90,105,120$ and 135 minutes $))$. Significant differences between the samples treated with firocoxib and flunixin $\left(R 1_{\text {Flun, }} R 1_{\text {Firo }}\right)$ could be seen at the highest stages of concentration.

The cumulative increase of the agent's concentration in case of flunixin led to a significant decrease of the median force of contractions. At the measurement times ZP5 and ZP6 (120 and 135 minutes), significant decreases of the samples $R I_{\text {Flun }}$ to the median values of force of the samples $R 1$ could be seen (ZP5: $P=0.0031, Z P 6: P<0.0001)$ and also in comparison to the samples $R 1_{\text {Firo }}$ at ZP5 $(P=0.0104)$ and ZP6 $(P<0.0001)$. Also a significant decrease over time could be seen within the samples of $R 1_{\text {Flun' }}$ from ZP1 to ZP5 $(P=0.0116)$, from $Z P 1$ to $Z P 6(P=0.0001)$, from $Z P 2$ to $Z P 6$ $(P=0.0001)$, from ZP3 to ZP5 $(P=0.0297)$, from ZP3 to ZP6 $(P<0.0001)$ and from ZP4 to ZP6 $(P=0.0018)$. The graphical depiction is shown in Figure 6.

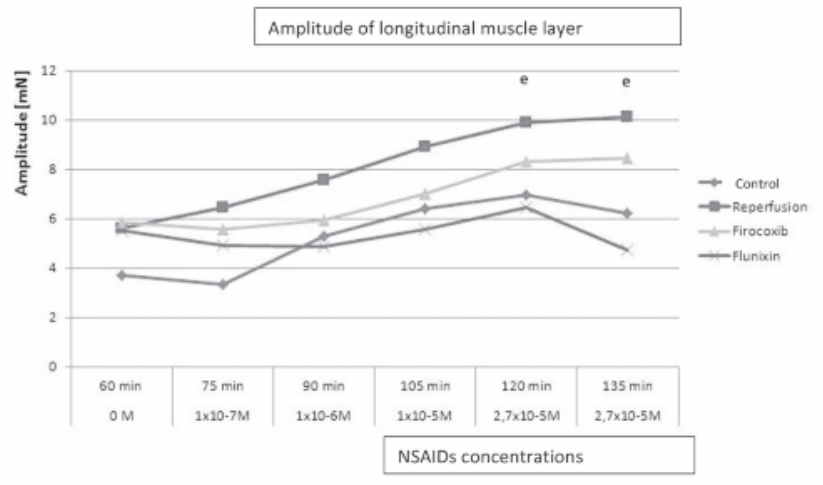

Fig. 5 Effect of the cumulative increase of the NSAID concentrations and the time on the amplitude of contractions of the various samples of longitudinal muscle. e = significant difference between R 1 from $Z P 1$ to $Z P 5$ and $Z P 6 ; f=$ significant difference between $R 1$ and $R 1_{\text {Flun }}$

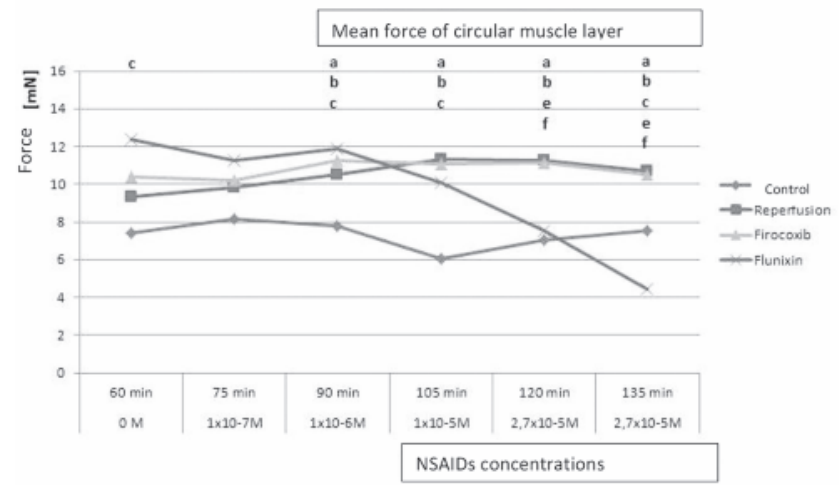

Fig. 6 Effect of the cumulative increase of the NSAID concentrations and the time on the median force of contractions of the various samples of circular muscle. $a=$ significant difference between $\mathrm{K} 1$ and $\mathrm{R} 1 ; \mathrm{b}=$ significant difference between $\mathrm{K} 1$ and $\mathrm{R} 1$ Firo; $c=$ significant difference between $\mathrm{K} 1$ and $R 1_{\text {Flun }} ; \mathrm{e}=$ significant difference between $R 1$ and $R 1_{\text {Flun }} ; f=$ significant difference between $R l_{\text {Firo }}$ and $R 1_{\text {Flun }}$
Summary of the first experiment (in vitro)

At the highest concentration stage flunixin, after in vitro application, showed a significantly inhibitory effect concerning contractility, whereas firocoxib achieved no significant effects. The damage through ischemia and reperfusion had a significantly increasing effect concerning contractility.

\section{Experiment 2: in vivo application of the agents}

Six of the probands included in the evaluation were treated with flunixin meglumine and five with firocoxib. The measurement of contractility of the five in vivo treated muscle samples took place subsequently to the measurements of the first experiment.

\section{Effects of the in vivo application on the longitudinal muscle}

Frequency

At both measurement times, the samples $\mathrm{R} 2_{\text {Flun }}$ and $\mathrm{K} 3_{\text {Flun }}$ showed significantly higher values for the frequency of contractions than the samples $\mathrm{R} 2_{\text {Firo }}$ and $\mathrm{K} 3_{\text {Firo. }}$. The graphical depiction is demonstrated in Figure 7.

\section{Amplitude}

Both samples, damaged through ischemia and reperfusion, with NSAID application (flunixin meglumine and firocoxib) showed a higher amplitude of contractions than the control samples without NSAIDs.

\section{Median force}

The samples treated in vivo with flunixin meglumine showed a significantly higher force in the samples damaged through ischemia and reperfusion in comparison to the samples $\mathrm{K} 2$ as well as to the samples $\mathrm{K}_{\text {Firo }}$ at both measurement times (ZP1: $P=0.0257, Z P 2: P=0.0301)$. Samples with firocoxib showed

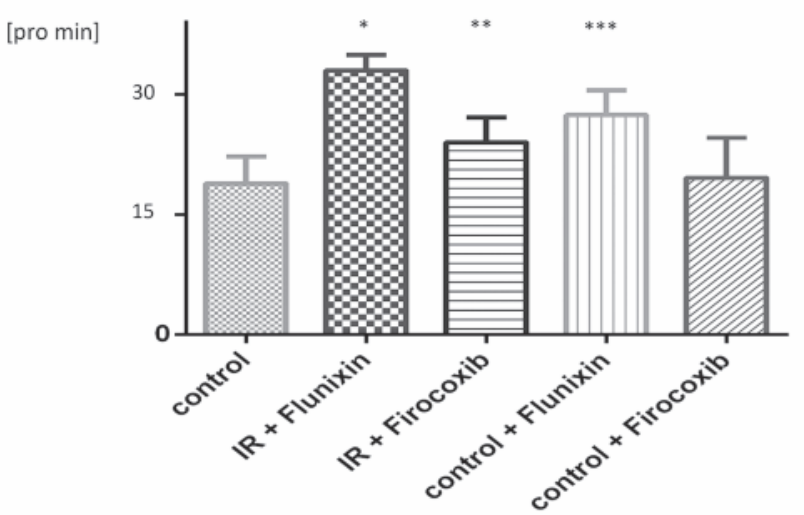

Fig 7 Frequency of longitudinal muscle contractions after treatment with NSAIDs in vivo. ${ }^{*}=$ significantly higher than control group, IR + Firocoxib, control + Firocoxib; ${ }^{* *}=$ significantly higher than control group; ${ }^{* * *}=$ significantly higher than control group, control + Firocoxib 
a significantly higher force in the damaged samples $R 2_{\text {Firo }}$ in comparison to the samples K2 (ZP1: $\mathrm{P}=0.0193, \mathrm{ZP} 2$ : $\mathrm{P}=0.0201)$ and $\mathrm{K} 3_{\text {Firo }}(\mathrm{ZP} 1: \mathrm{P}=0.0102, \mathrm{ZP} 2: \mathrm{P}=0.0112)$.

\section{Effects of the in vivo application on the circular muscle}

Frequency

The samples treated in vivo with flunixin meglumine showed a significantly higher frequency in the damaged samples $\mathrm{R} 2_{\text {Flun }}$ than the samples $\mathrm{K} 2$.

\section{Median force}

Effect of the intravenous treatment with flunixin: The samples treated in vivo with flunixin showed a significantly higher force of contractions in the circular muscle regarding the samples $R 2_{\text {Flun }}$ in comparison to the samples $K 2(Z P 1: P=0.0443$, $Z P 2: P=0.0423), R 2_{\text {Firo }}(Z P 1: P=0.0454, Z P 2: P=0.0487)$, $K 3_{\text {Flun }}(Z P 1: P=0.0320, Z P 2: P=0.0012)$ and $K 3_{\text {Firo }}(Z P 1$ : $P=0.0544, Z P 2: P=0.0538$ ) at both measurement times. The samples R2 flun showed a significantly higher median force at the point of the second measurement $(P=0.0106)$ than the samples $\mathrm{K} 3_{\text {Flun }}$.

\section{Summary of the second experiment (in vivo), Tables 1 and 2}

Regarding the longitudinal muscle strips, the in vivo application of flunixin led to a significant increase of the frequency in the damaged samples in comparison to the samples with firocoxib application and the control samples, whereas the amplitude of the longitudinal muscle strips was significantly increased in the damaged samples through application of firocoxib in comparison to the control group. The median force in the damaged samples after application of flunixin and firocoxib did not significantly differ but was higher than the median force of the unharmed control and the unharmed samples after application of flunixin and firocoxib.

In case of the circular muscle strips, at the point of the first measurement a significantly higher frequency of the damaged samples with flunixin could be seen in comparison to the control samples. Significant differences regarding the amplitude of all samples were not apparent. The median force was significantly higher in the R2Flun than in the other samples and the K3Flun showed the lowest median force.
Control samples in the first and second experiment - effects of the factor time

There were no significant differences regarding frequency, amplitude and median force of contractions between both control groups.

\section{Discussion}

The warm ischemia model was chosen for the presented study due to its similarity to the ischemic situation during a colic (Blikslager et al. 2008, Moore et al. 1995). This applied ischemia and reperfusion model led to detectable ischemia and reperfusion damage in form of a significant immigration of neutrophil granulocytes and oedema formation (Vente 2011). Damage by the experimental ischemia and reperfusion led to significant hypercontractility (probably contractility increase due to damage) in this study in comparison to unharmed samples of longitudinal and circular muscle layer in vitro. This assumption is supported by observations of increased contractility of the damaged intestinal segments in vivo in the presented study. The reasons for the increase in contractility through the damage after ischemia and reperfusion could be an upregulation of the COX isoforms, especially of the inducible COX-2, and resulting higher rates of synthesis of prostaglandins.

In the presented study also an effect of different NSAIDs on the intestinal contractility could be seen and an effect on the gastrointestinal functions due to $\mathrm{COX}$ inhibition through the NSAIDs is probable (van Hoogmoed et al. 2000).

Due to the results of both experiments, a toxicological effect of flunixin after in vitro application of the agent is assumed, by the authors, to be the most probable explanation of the significant inhibitory effect. Possibly, toxic pharmacological effects in case of the in vitro application of the agents occurred in the presented study, as also discussed in the work group of van Hoogmoed et al. (2002). Probably, higher agent concentrations were reached with in vitro application of the agents than after intravenous application. The high plasma protein bond of flunixin (up to $99 \%$ ) causes only approximately $1 \%$ to reach the effect area free in the cell. Therefore it is probable that after intravenous application of flunixin substantially lower tissue concentrations are reached than the highest that were chosen for in vitro application in this study. It is possible that this leads to an intoxication of the tissue

\begin{tabular}{lcc}
\hline Tab 1 & Tabular summary of the effects on the longitudinal muscle after in vivo application of NSAIDs & \\
\hline \multicolumn{1}{l}{ longitudinal } & flunixin & firocoxib \\
\hline frequency & increased (comparison: all samples excl. K3 Flun & increased (comparison: control) \\
amplitude & increased (comparison: control) & increased (comparison: control) \\
median force & increased (comparison: all unharmed samples) & increased (comparison: all unharmed samples) \\
\hline
\end{tabular}

\begin{tabular}{lcc}
\hline Tab 2 & Tabular summary of the effects on the circular muscle after in vivo application of NSAIDs \\
\hline circular & flunixin & firocoxib \\
\hline frequency & increased (comparison: control) & no effect \\
amplitude & no effect & no effect \\
median force & increased (comparison: control) & no effect
\end{tabular}


through the agents after in vitro application and therefore results in a different effect than after intravenous in vivo application. In order to verify a toxic tissue effect, actual tissue concentrations after in vivo application should be determined.

Due to the results of the presented study, it possibly can be assumed that, in case of intravenous application in therapeutic dosage, lower concentrations can be reached than those agent concentrations used in vitro and that, after in vitro application, there may have been an inhibition of the intestinal motility through a COX-1 independent mechanism (van Hoogmoed et al. 2002). The different results of the first (in vitro application of the agents) and the second part of the study (intravenous in vivo application of the NSAIDs) also allow the possibility of a direct interaction of the agents flunixin and firocoxib with the smooth muscle cells. Possibly, this interaction is also not COX-mediated. Also possible are effects of the different application possibilities of the NSAIDs: the in vitro application with direct tissue exposition and the intravenous in vivo application. The in vitro application results in direct tissue contact and, in case of the in vivo application, the flunixin is applied intravenously as injection solution and reaches the muscle cells via bloodstream. A toxic effect through direct tissue contact is possible. Also it has to be considered that drugs applied in vitro underlie no sorts of metabolism and that the physiological medium blood is replaced by a serum-like buffer solution, isotonically and ionically adapted to blood. Further it has to be assumed, that the samples of the second experiment were influenced by the previous first experiment. Here, a panenteritic process could have resulted due to manipulation and long surgery time. Therefore it is difficult to transfer results gained in vitro to in vivo circumstances and therapeutic doses. Through the metabolism missing in vitro, a prolonged tissue exposition with the drug at its highest concentration rate occurs. The effects of the COX inhibitors on local cellular mechanisms are not fully clarified and require further research.

In the presented study, different effects on both muscle layers took place, especially through in vitro application of flunixin. Indeed an inhibitory effect of flunixin in high concentrations $\left(1 \times 10^{-5} \mathrm{M}-2.7 \times 10^{-5} \mathrm{M}\right)$ occurred on the contractility (frequency, amplitude, median force) in both muscle layers, however the reduction of the contractility in the longitudinal muscle led to similar values as those of the untreated control, whereas a reduction of the contractility under the values of the control was seen and many samples showed no more spontaneous contractility. These differences could be evidence for generally different distribution of COX in the muscle layers (more in the circular than in the longitudinal muscle layer), different distribution of COX isoforms, temporally different upregulation of the COX isoforms or a different response, respectively reaction, to the corresponding synthesis products of the COX. Further, a different degree of the muscle (number of muscle fibres per area) has to be taken as a possibility for the varying reactions.

Due to missing effects after in vitro application of prostaglandin antagonists to muscle strips tied in organ baths, Menozzi et al. 2009 concluded that maybe there is no direct correlation between the effect of the NSAIDs and the influencing of the intestinal contractility through the effect of prostaglandin. Also the results of the presented study could indicate that the various COX isoforms play a varying role in the modulation of the motility of the small intestine. The results of this study concerning in vitro application of the agents could be a result of the different selectivity and therefore different inhibition of the COX isoforms through flunixin and firocoxib. The strong selective inhibition of COX-2 by firocoxib, however, does not seem to influence the contractility in vitro.

Other experiments with the COX-1 selective inhibitor SC-560 allowed the conclusion that the COX-1 isoform plays a more subordinate role in the regulation of the motility of the small intestine (Wallace 2000). However, it is assumed that COX-2 plays a role in the regulation of the gastrointestinal motility (Little et al. 2007b, Bernardini et al. 2006). The exact physiological function however is not clear, but is probably based on the coexpression of different prostaglandin synthases (Little et al. 2007b, Bernardini et al. 2006).

Concerning the in vitro measurements of contractility in this present study, the modulation of the contractility gives a different picture after intravenous in vivo application of the NSAIDs flunixin and firocoxib. Here, a significant increase of the contractility parameters could be seen after intravenous flunixin meglumine application. The reason could be the PGE2 reduction (PGE2 has inhibitory effects on the in vitro contractility of the intestine) through the flunixin-related inhibition of COX-1.

\section{Summary and clinical relevance}

In vitro application of flunixin resulted in inhibitory effects. Because the inhibitory effect only appeared in the highest agent concentration and also only when applied in vitro, a toxicological effect is probable. In vivo, these tissue concentrations in the dosage recommended by the manufacturer are probably never reached. Also, the direct contact with the tissue that occurred in case of the in vitro application and that is not applicable to the in vivo situation has to be considered as possible explanation for the results. After in vitro application, Firocoxib showed no significant differences in all three measured parameters (frequency, amplitude and median force) in the longitudinal muscle strips as well as the circular muscle strips to the untreated sample after ischemia and reperfusion.

After in vivo application of the agents, a different result became apparent. Here there seems to be an increasing effect through flunixin meglumine regarding contractility in the equine jejunum damaged through ischemia and reperfusion. These results are much closer to use in an actual equine colic patient due to the application form, the initial intravascular distribution in the body and the concentrations in the tissue, and therefore more applicable to the in vivo situation of a horse with colic. However, these results cannot just be extrapolated because in vitro only the contractility but not the directed motility could be measured.

It is questionable whether the hypercontractility shown in vitro after damage through ischemia and reperfusion leads to a motility disorder in vivo. The inhibitory effects after in vitro application of flunixin could not be comprehended after in vivo application. After in vivo application, no evidence of inhibitory 
effects on the measured parameters concerning the contractility of the small intestine through NSAID application could be found, therefore also no possible evidence for a participation of the effects of NSAID on a postoperative ileus. Further examinations must show whether other intestinal segments react similarly and whether different reactions occur after different damage. The results of the other study group that worked with the same sample material showed that the COX-1 expression was especially present in the circular muscle layer of the tunica muscularis, however, only very poor in the longitudinal muscle layer. The expression of COX-2 was substantially lower and could then be seen in the longitudinal muscle layer (Franz 2013). This is an interesting aspect when thinking about the consequences of COX inhibition using NSAIDs.

\section{Conclusions}

An in vitro study is always only partly appropriate to draw conclusions about the actual in vivo situation. However, an in vitro study is good for depicting certain effects and then gaining indications which make the in vivo situation more comprehensible and in order to then influence these specifically. This study gave indications that an influence on the contractility through the application of COX inhibitors is probable due to a toxicological effect after in vitro application and, after in vivo application, possibly due to the synthesis inhibition of prostaglandins acting inhibitory on contractility. The results of this study concerning the intravenous application of the agents allows the conclusion that none of the examined NSAIDs (flunixin and firocoxib) is clearly superior to the other and therefore the use of a special NSAID cannot clearly be recommended.

\section{Animal welfare statement}

For the experiments carried out in this study, a proposal for animal testing was submitted to, and approved by, the Lower Saxonian State Office for Consumer Protection and Food Safety (LAVES) pursuant to Section 8, Paragraph 1 of the Animal Welfare Act in accordance with Appendix 1 of the General Administrative Regulations of 9 September 2000 (Az: 33.9-42502-04-11/0572).

\section{References}

Adams S. B., Lamar C. H., Masty J. (1984) Motility of the distal portion of the jejunum and pelvic flexure in ponies: Effect of six drugs. Am. J. Vet. Res. 45, 795-799
Blikslager A. T. (2008) Life in the gut without oxygen: adaptive mechanisms and inflammatory bowel disease. Gastroenterology $134,346-348$

Bernardini N., Colucci R., Mattii L., Segnani C., Fornai M., De Giorgio R., Barbara G., Castagna M., Nardini V., Dolfi A., Del Tacca M., Blandizzi C. (2006) Constitutive expression of cyclooxygenase-2 in the neuromuscular compartment of normal human colon. Neurogastroenterol. Motil. 18, 654-662

Franz S. (2013) Effekt selektiver und nicht selektiver nichtsteroidaler Antiphlogistika auf die Entzündungsreaktion von durch Ischämie und Reperfusion geschädigtem equinen Jejunum. Diss. Med. Vet. Hannover

Hardy J., Rakestraw P. C. (2002) Postoperative management for colics. Clin. Techniques in Equine Practice 1, 188-197

Lester G. D., Merritt A. M., Neuwirth L., Vetro-Widenhouse T., Steible C., Rice B. (1998b) Effect of $\alpha 2$-adrenergic, cholinergic, and nonsteroidal anti-inflammatory drugs on myoelectric activity of ileum, cecum, and right ventral colon and on cecal emptying of radiolabeled markers in clinically normal ponies. Am. J. Vet. Res. 59, 320-327

Little D., Jones S. L., Blikslager A. T. (2007b) Cyclooxygenase (COX) inhibitors and the intestine. J. Vet. Intern. Med. 21, 367-377

Lowe J. E., Sellers A. F., Brondum J. (1980) Equine pelvic flexure impaction. A model used to evaluate motor events and compare drug response. Cornell Vet. 70, 401-412

Menozzi A., Pozzoli C., Poli E., Dacasto M., Giantin M., Lopparelli R. M., Passeri B., Zullian C., Gobbetti T., Bertini S. (2009) Effects of nonselective and selective cyclooxygenase inhibitors on small intestinal motility in the horse. Res. Vet. Sci. 86, 129-135

Moore R. M., Muir W. W., Granger D. N. (1995) Mechanisms of gastrointestinal ischemia-reperfusion injury and potential therapeutic interventions: A review and its implications in the horse. J. Vet. Intern. Med. 9, 115-132

Roger T., Ruckebusch Y. (1987) Pharmacological modulation of postprandial colonic motor activity in the pony. J. Vet. Pharmacol. Ther. 10, 273-282

Ross M. W., Martin B. B., Donawick W. J. (1985) Cecal perforation in the horse. J. Am. Vet. Med. Assoc. 187, 249-253

van Hoogmoed L., Rakestraw P. C., Snyder J. R., Harmon F. A. (1999) In vitro effects of nonsteroidal anti-inflammatory agents and prostaglandins $12, E 2$, and F2alpha on contractility of taenia of the large colon of horses. Am. J. Vet. Res. 60, 1004-1009

van Hoogmoed L. M., Snyder J. R., Harmon F. (2000) In vitro investigation of the effect of prostaglandins and nonsteroidal antiinflammatory drugs on contractile activity of the equine smooth muscle of the dorsal colon, ventral colon, and pelvic flexure. Am. J. Vet. Res. 61, 1259-1266

van Hoogmoed L. M., Snyder J. R., Harmon F. A. (2002) In vitro investigation of the effects of cyclooxygenase- 2 inhibitors on contractile activity of the equine dorsal and ventral colon. Am. J. Vet. Res. 63, 1496-1500

Vente C. (2011) Intestinale Entzündungsreaktionen während Ischämie und Reperfusion im Jejunum des Pferdes unter besonderer Berücksichtigung der eosinophilen und neutrophilen Granulozyten. Diss. Med. Vet. Hannover

Wallace J. L., Mcknight W., Reuter B. K., Vergnolle N. (2000) NSAIDinduced gastric damage in rats: requirement for inhibition of both cyclooxygenase 1 and 2. Gastroenterology 119, 706-714 